\title{
APROXIMACIÓN AL COSTE DEL TRATAMIENTO MÉDICO DE LA HIPERPLASIA BENIGNA DE PRÓSTATA EN LA COMUNIDAD FORAL DE NAVARRA
}

\author{
V. GRASA LANAU*, J.Á. CUESTA ALCALÁ*, A. IPIENS AZNAR*, I. ANSA ERICE**, \\ J. ALDAVE VILLANUEVA*
}

*Servicio de Urología. Hospital de Navarra. Pamplona (Navarra).

**Servicio de Prestaciones Farmacéuticas, SNS-O.

Actas Urol Esp. 28 (4): 255-261, 2004

\section{RESUMEN}

APROXIMACIÓN AL COSTE DEL TRATAMIENTO MÉDICO DE LA HIPERPLASIA BENIGNA DE PRÓSTATA EN LA COMUNIDAD FORAL DE NAVARRA

Se estudia el coste y evolución del tratamiento médico ambulatorio de los pacientes afectos de clínica de prostatismo sugestiva de hiperplasia benigna de próstata en la Comunidad Foral de Navarra en el periodo $1998 / 2002$.

El número estimado de pacientes en tratamiento se incrementa cada año, llegando al 10\% de la población masculina mayor de 50 años en el 2002, con un coste para el ente gestor de $2.557 .236 €$, el 2,4\% del gasto médico farmacéutico extrahospitalario total (106,6 millones €).

En dicho periodo, la tamsulosina triplica su uso y dobla el coste hasta los $807.467 €(31,5 \%$ del total) mientras que el resto de alfa-bloqueantes, con la excepción de la doxazosina, prácticamente se estacionan. La fitoterapia baja su utilización en casi un tercio y el coste en un 50\%. El finasteride mantiene una línea ascendente con una cuota de mercado actual del 15\%. La introducción de los precios de referencia el año 2001 disminuye inicialmente el gasto, pero esta reducción queda sin efecto ya el año siguiente ante la incorporación de nuevos pacientes en tratamiento.

En el mismo periodo, la cirugía por adenoma de próstata (cerrada y abierta) pasó de 265 y 117 pacientes en 1998 a 195 y 75, respectivamente, en el año 2002 (-30\%).

PALABRAS CLAVE: Hiperplasia benigna de próstata. Tratamiento médico. Coste.

\section{ABSTRACT \\ ESTIMATION OF THE COST IN THE MEDICAL TREATMENT OF BENIGN PROSTATIC HYPERPLASIA IN NAVARRE, SPAIN}

We evaluate the cost and trends in the medical treatment of out patients suffering from lower urinary tract symptoms suggestive of clinical benign prostatic hyperplasia in Navarre (Spain) between 1998/2002. The estimated number of patients increased each year, to $10 \%$ of the male population over the age of 50 in 2002, with a cost of $€ 2.557 .236$ equivalent to $2.4 \%$ of the total drug expenditure spending of out patients ( $€ 106.6$ million). The use of tamsulosin tripled and the cost doubled to $€ 807.467$ (31.5\%) of the total), while the rest of alpha-blockers, wit the exception of doxazosin, was stationary. Phytotherapy decreased by a third and finasteride follows a slow upward trend. The introduction of reference prices set by the Health Department in 2001 to reduce medical budget, led to an initial decrease in cost, offsetted in the following year due to the incorporation of new patients.

In this period, surgery for prostate adenoma diminished from 382 patients in 1998 to 270 in 2002 $(-30 \%)$.

KEY WORDS: Benign prostatic hyperplasia. Treatment. Medical therapy. Economics. Cost. 
$\mathrm{L}$ a hiperplasia benigna de próstata (HBP) constituye una de las patologías más frecuentes del varón a partir de la sexta década de la vida, repercute muy negativamente en la Calidad de Vida $(\mathrm{CdV})$ por su clínica de síntomas del tracto urinario inferior (STUI) o prostatismo y consume una parte significativa de los recursos sanitarios disponibles. Según una reciente revisión ${ }^{1}$, más de un tercio de los pacientes que consultan al urólogo por este proceso reciben tratamiento médico y el 38\% necesitará algún tipo de procedimiento invasivo. La HBP es un problema sanitario en los países desarrollados, si se incluye bajo este epígrafe de acuerdo con Baena y cols. ${ }^{2}$ a cualquier proceso patológico de alta incidencia, carga económica y severa repercusión en la $\mathrm{CdV}$ del enfermo. La ausencia de una definición uniformemente aceptada para la HBP clínica hace que su prevalencia real e historia natural sean mal conocidas ${ }^{3}$. Tampoco se conoce la relación costo/eficacia de las diversas opciones terapéuticas. Todo ello dificulta las estrategias conjuntas y uniformes de actuación y confiere a la HBP una extraordinaria variabilidad clínica en lo que se refiere al tratamiento.

La HBP para algunos autores ${ }^{4}$ es ya la primera causa de consulta ambulatoria al especialista. Dado que la edad es el factor univariante que más influencia su aparición y desarrollo, esta tendencia creciente debe ir en aumento en los próximos años por el envejecimiento de la población, aumento en la esperanza de vida e incremento de la frecuentación médica en demanda de una mejor CdV. Con estas premisas, el conocimiento de su coste y posible evolución, adquiere un interés evidente a todos los efectos.

La determinación del coste real del tratamiento de la HBP es sumamente difícil, dada la complejidad de los parámetros a estudiar, los cuales incluyen la ya citada variabilidad en su definición y tratamiento, junto a la adecuada valoración de los costes indirectos y los no tangibles ${ }^{2}$.

Sin embargo, sí es posible un acercamiento al problema del coste mediante la cuantificación de lo que éste supone para la entidad o sistema sanitario que cubre la prestación en aspectos concretos del mismo, como es el tratamiento médico. Esto es especialmente posible, pese a todas las limitaciones citadas, en una Comuni- dad Autónoma como la Navarra (CFN), de extensión y demografía reducidas, con unos más que aceptables medios de registro y contabilidad y cuyo ente gestor, el Servicio Navarro de SaludOsasunbidea (SNS-O), realiza una asistencia pública con prestaciones prácticamente universales en el tratamiento farmacológico de esta patología a la población del referido territorio.

En este estudio se pretende conocer el coste del tratamiento médico extrahospitalario de los pacientes afectos de clínica de prostatismo, posiblemente causada por HBP, para el SNS-O y analizar las tendencias en el consumo de los diversos grupos terapéuticos a lo largo de los últimos cinco años en la CFN, sin enjuiciar en ningún momento la idoneidad del mismo o su eficacia. También se estudia el número de actuaciones quirúrgicas realizadas por $\mathrm{HBP}$ clínica. Aunque una primera revisión del tema hasta el año 2001 ya fue comunicada ${ }^{5}$, aquí se expondrán y analizarán estos aspectos con mayor detalle incluyendo los datos referidos al año 2002.

\section{MATERIAL Y MÉTODO}

Se investiga el gasto farmacológico que el tratamiento médico extrahospitalario de la HBP clínica y el prostatismo, supuso para la Sanidad Pública Navarra, desglosándolo por principios terapéuticos los años 1998, 2001 y 2002.

El estudio se inició el año 1998, pues éste era el primero en el que se disponía de una contabilidad totalmente informatizada y plenamente fiable sobre el consumo farmacéutico, tanto en gasto como en dispensación. El último estudiado fue el 2002, con el fin de disponer de cifras completas para todo el año. Ante la cantidad de datos numéricos, se prescindió de los años 1999 y 2000 con el fin de simplificar los cuadros, ya que su inclusión no aportaba cambios en las tendencias de gasto esbozadas con los años que se han valorado ni alteraban las conclusiones.

Los datos demográficos de Navarra proceden del Instituto Nacional de Estadística ${ }^{6}$ (Tabla I). El Servicio de Prestaciones Sanitarias del SNS-O, aportó las cifras relativas al total del consumo farmacéutico extrahospitalario (Tabla II) y el número de envases facturados para la clínica de prostatismo, desglosados por nombres comerciales y principios activos junto a su coste para el 


\section{TABLA I}

PACIENTES PORTADORES DE CANCER PROSTATICO

\begin{tabular}{|c|c|c|c|}
\hline $\begin{array}{c}10.391 \\
\mathrm{Km}^{2}\end{array}$ & $\begin{array}{c}\text { Total } \\
\text { habitantes }\end{array}$ & $\begin{array}{c}\text { Habitantes } \\
>50 \text { años }\end{array}$ & $\begin{array}{c}\text { Habitantes } \\
\text { varones }>50 \text { años }\end{array}$ \\
\hline 1998 & 530.819 & 181.716 & 83.835 \\
\hline 2001 & 556.263 & 192.188 & 88.962 \\
\hline 2002 & 569.628 & 192.613 & 89.026 \\
\hline
\end{tabular}

TABLA II

COSTE POR GRUPOS TERAPÉUTICOS EN 2002

CONSUMO FARMACÉUTICO EXTRAHOSPITALARIO EN LA CFN TOTAL APROX. 106,6 MILLONES DE $€$

\begin{tabular}{|r|l|r|}
\hline & Grupo terapéutico & Coste en $€$ \\
\hline 1 & Hipotensores & 10.175 .680 \\
\hline 2 & Antidepresivos & 8.050 .184 \\
\hline 3 & Antiúlcera péptica & 7.859 .228 \\
\hline 4 & Hipolopemiantes & 7.583 .823 \\
\hline 5 & Broncodilatadores & 6.925 .498 \\
\hline 6 & Tto miocardio & 4.696 .334 \\
\hline 7 & AINE & 4.457 .022 \\
\hline 8 & Antibióticos & 4.373 .509 \\
\hline 9 & Neurolépticos & 3.673 .290 \\
\hline 10 & Antiagregantes plaquetarios & 3.394 .308 \\
\hline 11 & Analgésicos & 3.287 .671 \\
\hline 12 & Hormonoterapia antineoplásica & 2.955 .261 \\
\hline 13 & HBP & 2.557 .236 \\
\hline 14 & Antiepilépticos & 2.339 .363 \\
\hline
\end{tabular}

sistema en cada uno de los años señalados (Tablas III y IV).

Al mismo tiempo se obtuvieron los datos numéricos de los procedimientos quirúrgicos realizados sobre la HBP en todos los Hospitales Públicos de la Red Sanitaria Foral y Centros Concertados. Estos datos se agruparon en dos apartados, uno de "cirugía abierta" y otro de "cirugia endoscópica", incluyendo en este grupo las trígono-cervico-prostatotomías (TCP), por su escaso peso numérico y con el fin de homogeneizar las series. Los procedimientos mínimamente invasivos no fueron considerados, ya que su práctica es puramente anecdótica hasta el momento en nuestro medio.
TABLA III

№ DE ENVASES VENDIDOS POR PRINCIPIO ACTIVO

\begin{tabular}{|l|c|c|c|}
\hline & $\mathbf{1 9 9 8}$ & $\mathbf{2 0 0 1}$ & $\mathbf{2 0 0 2}$ \\
\hline Doxazosina & 21.774 & 36.752 & 40.144 \\
\hline Tamsulosina & 8.682 & 22.427 & 27.119 \\
\hline Otros alfa & 13.577 & 16.281 & 16.695 \\
\hline Fitoterapia & 26.103 & 18.977 & 18.558 \\
\hline Finasteride & 11.026 & 14.928 & 17.459 \\
\hline Total & $\mathbf{8 1 . 1 6 2}$ & $\mathbf{1 0 9 . 3 6 5}$ & $\mathbf{1 1 9 . 9 7 5}$ \\
\hline
\end{tabular}

TABLA IV

GASTO EN $€$ POR PRINCIPIO ACTIVO

\begin{tabular}{|l|c|c|c||}
\hline & $\mathbf{1 9 9 8}$ & $\mathbf{2 0 0 1}$ & $\mathbf{2 0 0 2}$ \\
\hline Doxazosina & 572.638 & 634.484 & 708.709 \\
\hline Tamsulosina & 428.854 & 667.673 & 807.467 \\
\hline Otros alfa & 421.164 & 328.126 & 336.055 \\
\hline Fitoterapia & 413.066 & 205.466 & 210.217 \\
\hline Finasteride & 518.052 & 423.115 & 494.788 \\
\hline Total & $\mathbf{2 . 3 5 3 . 7 7 4}$ & $\mathbf{2 . 2 5 8 . 8 6 4}$ & $\mathbf{2 . 5 5 7 . 2 3 6}$ \\
\hline
\end{tabular}

El número de pacientes en tratamiento se estimó a partir del número de envases vendidos para cada principio activo, aceptando que la administración correspondía a la posología recomendada y que cada paciente tomaba un solo fármaco. Este método evidentemente no es exacto, con seguridad tiene errores y puede ser criticable, pero permite una aproximación al problema y con los medios disponibles por el momento, es la única manera de plantear el cálculo aproximado de pacientes en tratamiento. En el apartado de discusión se abordará este punto con más detalle.

No se han incluido en el estudio los pacientes en tratamiento con prazosín, ya que se ha considerado que en la CFN son muy pocos los que lo utilizan con fines urológicos y se evita así una posible fuente de error.

\section{RESULTADOS}

De acuerdo con el cálculo seguido, el número de pacientes estimados en tratamiento médico para la HBP y STUI en la CFN, fue de 5.729 sobre un total de 83.835 varones mayores de 50 años $(6,8 \%)$ el año 1998 , cifra que sube a 8.912 sobre 89.026 (10\%) en el 2002 (Tabla V). 
TABLA V

№ ESTIMADO DE PACIENTES EN TRATAMIENTO

\begin{tabular}{|l|c|c|c|}
\hline & $\mathbf{1 9 9 8}$ & $\mathbf{2 0 0 1}$ & $\mathbf{2 0 0 2}$ \\
\hline Doxazosina & 1.644 & 2.775 & 3.031 \\
\hline Tamsulosina & 713 & 1.844 & 2.230 \\
\hline Otros alfa & 995 & 1.194 & 1.224 \\
\hline Fitoterapia & 1.531 & 1.113 & 1.088 \\
\hline Finasteride & 846 & 1.145 & 1.339 \\
\hline Total & $\mathbf{5 . 7 2 9}$ & $\mathbf{8 . 0 7 1}$ & $\mathbf{8 . 9 1 2}$ \\
\hline
\end{tabular}

Por grupos terapéuticos, el tratamiento médico de la HBP ocupa el lugar decimotercero después de la hormonoterapia antineoplásica en la que se incluye el carcinoma de próstata. El costo para la Sanidad Navarra del conjunto de fármacos incluidos para la HBP a lo largo del año 2002 fue de 2.557.236 €. Dado que el total del gasto médico farmacéutico extrahospitalario el mismo año ascendió a 106,6 millones de $€$, el tratamiento médico de la HBP así calculado supone el $2,39 \%$ del total.

El grupo farmacológico más utilizado es el de los alfa-bloqueantes y dentro de estos la doxazosina. Sin embargo, el gasto en tamsulosina es mayor y con tendencia creciente (Figs. 1 y 2). El uso de la fitoterapia está claramente en descenso y su coste se ha estabilizado en el último lugar tras el grupo "otros alfa-bloqueantes", mientras que el finasteride presenta una línea de progresión pequeña pero sostenida, con un coste intermedio. Hay que señalar también la "dispersión"

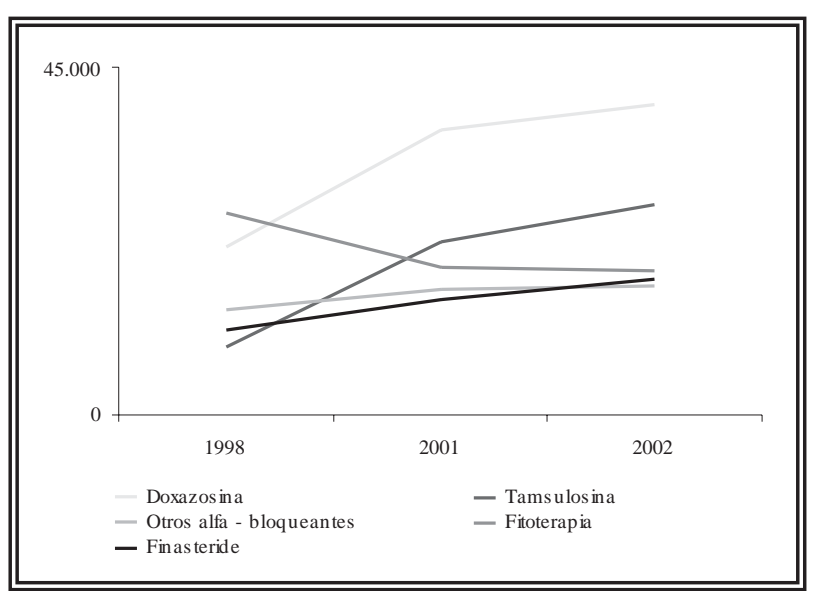

FIGURA 1. Evolución del número de envases financiados por el SNS-O en el periodo 1998/2002.

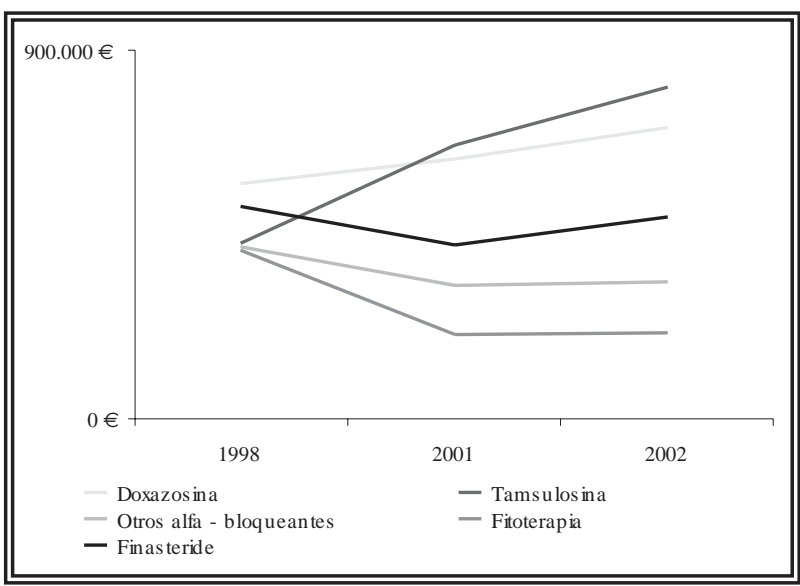

FIGURA 2. Evolución del gasto del tratamiento del prostatismo según los diversos grupos farmacológicos.

que experimenta el coste, pues si en 1998 oscilaba entre un máximo de 572.638 (doxazosina) y un mínimo de $413.066 €$ (fitoterapia), el 2002 varía entre 807.467 (tamsulosina) y $210.217 €$ (fitoterapia).

Mientras tanto, el tratamiento invasivo (cirugía abierta y endoscópica) bajó de 382 pacientes en 1998 a 265 en el año 2001 y pasó a 270 en el 2002 (Fig. 3).

\section{DISCUSIÓN}

El cuadro clínico producido por la HBP y denominado prostatismo o síntomas del cuadro urinario inferior (STUI) de acuerdo con la terminología más reciente, afecta al menos al $40 \%$ de los varones mayores de 60 años $^{7}$. Aproximadamente un tercio de éstos recibirán tratamiento médico aun-

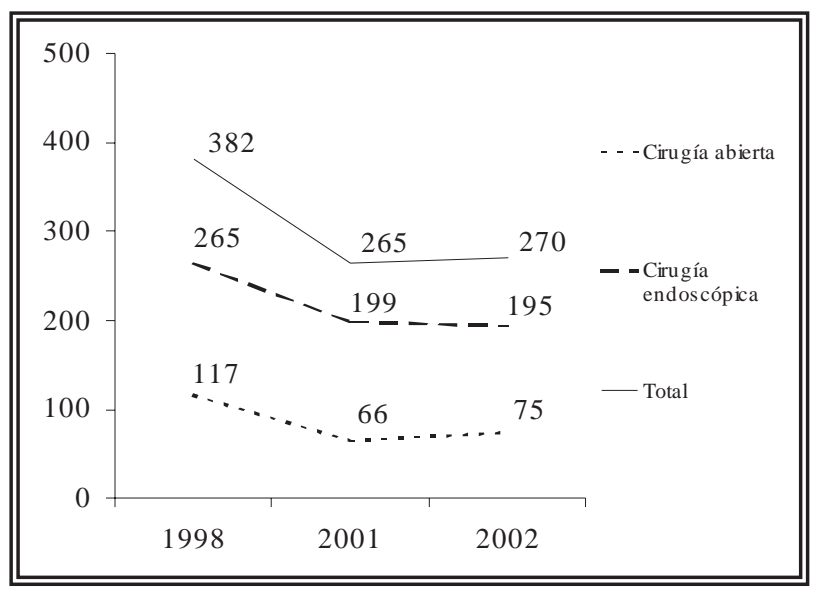

FIGURA 3. Número de cirugias por HBP desglosadas en abiertas, cerradas y suma de ambas, realizadas en la red pública entre 1998 y 2002. 
que las variaciones son muy amplias dependiendo de los países y sistemas sanitarios ${ }^{8}$. La cuantificación real del gasto total que esta patología supone es desconocida por su complejidad, lo que explica en parte la escasez de trabajos a este respecto y los frecuentes defectos metodológicos que suelen presentar la mayoria ${ }^{2}$, defectos de los que no se exime el nuestro. No obstante, creemos que ofrece al menos una aproximación a este problema en nuestro medio, problema que por sus dimensiones tiene y va a tener repercusiones no sólo sanitarias, sino también sociales, económicas y políticas en los próximos años.

Un estudio prospectivo de mercado señala que en el mundo desarrollado el año 2006, al menos 115 millones de varones mayores de 50 años necesitarán algún tipo de tratamiento para la HBP, con un coste total estimado de cerca de diez mil millones de dólares ${ }^{9}$.

Conscientes de estas dificultades y de la imposibilidad de abordar el problema en su totalidad, el estudio se ha centrado y limitado a conocer la carga o impacto que en la sanidad pública de una comunidad pequeña como la nuestra puede tener el tratamiento médico de la HBP y prostatismo, prescindiendo de la aportación realizada por el propio enfermo en función de su tipo de afiliación al sistema de Seguridad Social, ni tampoco la de aquellos pacientes incluidos en el censo pero que adquieren los fármacos al margen de la cobertura sanitaria. Dadas las características sociológicas de la población estudiada, pensamos que este último factor debe ser muy poco relevante.

Aún así, existen otras evidentes fuentes de error, como es el hecho bien conocido de que no todos los pacientes con clínica de prostatismo, presentan HBP clínica, pese a que la HBP sea el proceso que más frecuentemente la produce. Tampoco la única indicación de alguno de los preparados utilizados es la HBP, como sucede con la fitoterapia. El problema mayor a este respecto lo plantean los pacientes hipertensos y con prostatismo tratados con doxazosina para ambas patologías. Este grupo puede ser aún significativo en nuestra población aunque el uso de la doxazosina en la HTA debería disminuir de acuerdo con las actuales guías clínicas ${ }^{10} \mathrm{y}$ al margen del posible riesgo de afectación ventricu- lar por apoptosis del cardiomiocito, según se ha descrito in vitro tanto con la doxazosina como con el prazosín, pero no así con la terazosina ${ }^{11}$. Por otra parte, ya en el año 2000 el comité de expertos recomendaba tajantemente tratar por separado e independientemente la HTA y el prostatismo si ambos se presentaban asociados ${ }^{12}$, aunque quizás esta norma no se siga todavía escrupulosamente en la práctica clínica.

Otra posible fuente de error reside en la imposibilidad de conocer y distinguir los pacientes en tratamiento bajo régimen de asociación de fármacos. Este grupo puede no ser despreciable y su número ir en aumento en el caso de la asociación alfa-bloqueante con finasteride, tal y como se ha recomendado recientemente ${ }^{13}$. Tampoco es excepcional el caso de aquellos enfermos que no abandonan la fitoterapia al iniciar tratamiento con alfa-bloqueantes.

Por todo lo expuesto, los datos obtenidos por extrapolación referidos al número de pacientes en tratamiento deben tomarse con reservas, en sentido meramente orientativo y referido a clínica de STUI, aunque la mayoría de ellos presentarán HBP. Pese a ello, las cifras son concordantes y se deben aproximar a la realidad de la población estudiada.

Del estudio se aprecia que en el periodo $1998 / 2002$, el número de varones mayores de 50 años se incrementa en un $6,2 \%$ y el de pacientes en tratamiento pasa de 5.729 a 8.912, con un aumento del 55,6\%. Esto supondría que en el último año valorado, uno de cada diez varones mayores de 50 años estaría siendo sometido a tratamiento médico por STUI en nuestra comunidad, cifra aún por debajo de la comunicada en un reciente estudio en Austria ${ }^{8}$ y que indica que estamos lejos de tocar techo y queda amplio margen de crecimiento, tal como se aprecia en la evolución de los gráficos.

Por el contrario, el gasto pasa de 2.353.774 € a 2.557.236 €, con un crecimiento de "sólo" el $8,6 \%$. Una explicación a esta desproporción entre el incremento de pacientes y evolución del gasto, puede deberse al descenso del coste farmacéutico en el año 2001, coincidiendo con la introducción de los denominados "Precios de Referencia" que permitió tratar a más pacientes con menos gasto. Se deberá esperar algún año más para 
poder valorar si esa medida fue efectiva, pues tan sólo un año después (2002), el gasto total se volvía a situar claramente por encima del de 1998 (Fig. 4). No estamos en condiciones de afirmar si la subida se debe tan sólo a los pacientes nuevos incorporados o es por trasvase en el tratamiento a preparados de más alto precio.

La doxazosina experimenta un incremento en su uso, sin que el mismo apenas se traduzca en el gasto, al contrario de lo que sucede con la tamsulosina, cuyo aumento en la dispensación es paralelo al coste. El resto de alfa-bloqueantes presentan un mínimo crecimiento con el coste estabilizado. La fitoterapia, cuya utilización se estanca el último año después de una gran caída, representa el 8,2\% del gasto total en 2002 mientras que era el $17,5 \%$ en 1998.

Se acepta unánimemente que la carga económica que supone el tratamiento de la HBP es una partida significativa de cualquier presupuesto sanitario. Esto es evidente en nuestro caso, donde los 2.557.236 € empleados en su tratamiento, o al menos englobados por la administración en ese subtítulo, suponen el 2,39\% del total del gasto farmacológico extrahospitalario y ocupa por su cuantía el lugar decimotercero. Esta es una posición muy relevante, máxime si se considera que se trata de una patología que sólo afecta a un sexo y lo hace en un grupo de edad determinado.

Respecto al tratamiento invasivo de la HBP, tanto mediante cirugía abierta o endoscópica, el descenso entre los años 1998 y 2001 es evidente

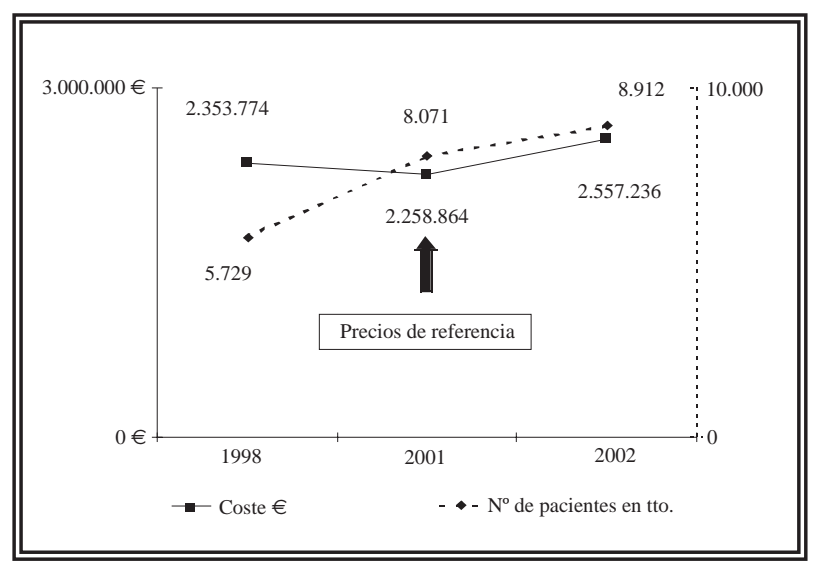

FIGURA 4. Número de pacientes en tratamiento y gasto médico en el periodo estudiado. La flecha señala la introducción de los denominados precios de referencia. y marcado, especialmente si se considera el incremento de población mayor de 50 años experimentado en el referido periodo. Se confirma así la tendencia ya descrita y conocida ${ }^{14}$ debida, entre otras razones, a la eficacia de los tratamientos farmacológicos actuales ${ }^{15}$.

\section{CONCLUSIONES}

En el periodo 1998/2001, la proporción de varones mayores de 50 años en tratamiento médico por STUI probablemente causados por HBP se incrementa substancialmente, pasando de acuerdo con el estudio realizado del $6,8 \%$ al $10 \%$, cifra aún por debajo de otros países europeos.

El crecimiento del consumo de fármacos para la HBP no es homogéneo. Mientras aumenta significativamente, con su obvia repercusión en los costes, la tamsulosina, los alfa-bloqueantes no uro-selectivos disminuyen con la excepción de la doxazosina, aunque la repercusión en el gasto es poco relevante. La fitoterapia experimenta un claro descenso al inicio del estudio, para estabilizarse el último año y el finasteride mantiene su cuota de mercado en el $15 \%$ de los pacientes en tratamiento y $20 \%$ del gasto, con tendencia sostenida al alza.

La introducción de los precios de referencia el año 2001 produce una inflexión momentánea en el gasto farmacéutico, cuya efectividad a corto y medio plazo está por confirmar.

La cirugía de la HBP se ha reducido significativamente en los últimos años, aunque este descenso podría haber llegado a su límite con las modalidades terapéuticas disponibles. Si éstas no cambian, el aumento progresivo de la población mayor de 50 años puede llevarla nuevamente al alza.

Se deben desarrollar programas de investigación que permitan conocer la relación costo-eficacia de las distintas modalidades terapéuticas, en línea con alguna iniciativa ya propuesta ${ }^{16}$. Esto exige un mejor conocimiento, entre otros, del número real de pacientes en tratamiento.

\section{REFERENCIAS}

1. WILT TJ.: Treatment options for benign prostatic hyperplasia. BMJ 2002; 324: 1047-1048.

2. BAENA GONZÁLEZ V, ARELLANO LUCENA J, BURGOS RODRÍGUEZ R.: Las enfermedades prostáticas: un problema sanitario. Jano 2001; 2: 5-12. 
3. MORENO SIERRA J, FERNÁNDEZ PÉREZ C.: Caracterización clínica y epidemiológica de los síntomas del tracto urinario inferior relacionados con la hiperplasia benigna de próstata en la Comunidad de Madrid. Cambio a los dos años en hombres asintomáticos. Madrid. Editorial EDIMSA 2002: 12-13.

4. CHICHARRO JA, BURGOS R.: Epidemiología de la hiperplasia benigna de próstata. Medicine 1997; 7: 3-8.

5. GRASA V, IPIENS A, ALDAVE J y cols.: Impacto del tratamiento de la hiperplasia benigna de próstata en la Comunidad Foral de Navarra. Actas Urol Esp 2003; 27 (4) (sup): C40, 34.

6. INSTITUTO NACIONAL DE ESTADÍSTICA. http://www.ine.es

7. DE LA ROSETTE J, SCHOOT DKE, DEBRUYNE FMJ. Recent developments in guidelines on benign prostatic hyperplasia. Cur Op Urol 2002; 12: 3-6.

8. TEMMPL C, BRÖSSNER C, SCHATZL G et al. The natural history of lower urinary tract symptoms over five years. Eur Urol 2003; 43: 374-380.

9. Benign Prostatic Hyperplasia: Global market analysis of treatment \& disease management implications. Report 1212A, 2002. http://www.thetareports.com

10. CHOBANIAN TJ and experts panel.: The seventh report of the joint national committee on prevention, detection, evaluation and treatment of high blood pressure: the JNC seventh report. JAMA 2003; 289: 2560-2572.
11. GONZÁLEZ-JUANATAY JR, IGLESIAS MJ, ALCAIDE C y cols.: Doxazosin induces apoptosis in cardiomyocites cultured in vitro by a mechanism that is independent of $\alpha_{1}$-adrenergic blockade. Circulation 2003; 107: 127-131.

12. CHATELAIN C, DENIS L, FOO KT, KHOURY S, MCCONNELL, editors.: Treatment recommendations. $5^{\text {th }}$ International Consultation on BPH, WHO 2000: 530-532.

13. MCCONNELL JD for the MTOPS steering committee: The long-term effects of medical therapy on the progression of BPH: results from the MTOPS trial. J Urol 2002; 167 (sup): 1042.

14. VICENTE J.: Tratamiento de la HBP, realidades presentes y perspectivas futuras. Actas Urol Esp 2002; 26: 481-490.

15. IPIENS A, GRASA V, CUESTA JA, ARRONDO JL.: Disfunción sexual en el tratamiento de la hiperplasia benigna de próstata. Arch Esp Urol (en prensa).

16. MCDONELL J, BUSSCHBACH J, KOK E et al.: Lower urinary tract symptoms suggestive of benign prostatic obstruction-triumph: health-economical analysis. Eur Urol 2001; 39 (sup 3): 37-41.

Dr. V. Grasa Lanau

Servicio de Urología. Hospital de Navarra

$\mathrm{C} /$ Irunlarrea, $\mathrm{s} / \mathrm{n}$

31008 Pamplona (Navarra)

(Trabajo recibido el 17 octubre de 2003) 